\title{
Receptor Activation Process
}

National Cancer Institute

\section{Source}

National Cancer Institute. Receptor Activation Process. NCI Thesaurus. Code C40702.

Any intercellular signaling process in which a transmembrane or intracellular receptor in the responding cell binds to a cognate ligand and initiates an intracellular response. Receptor activation is involved in numerous processes, including metabolism, immune response, sexual development, nervous system function, and regulation of circadian rhythms, mood and behavior. 